\title{
An Analysis of the Pattern of Adaptive Emotion Regulation Associated with Low Paranoid Ideation in Healthy and Clinical Samples
}

\author{
Martin F. Wittkamp ${ }^{1}$ (D) Katarina Krkovic ${ }^{1}$ (D) $\cdot$ Tania M. Lincoln $^{1}$ (D)
}

Accepted: 21 October 2020 / Published online: 11 November 2020

(c) The Author(s) 2020

\begin{abstract}
Background Research on emotion regulation and paranoid ideation has mostly focused on isolated regulation strategies and has remained largely inconclusive. According to the emotion regulation model by Berking and Whitley (in: Affect Regulation Training, Springer, New York 2014) successful modification or acceptance/tolerance of emotions requires an adequate comprehension (awareness, clarity, understanding) of emotions and adequate self-support.

Method Building on this model, we investigated whether comprehension and self-support strengthen the negative association between modification and acceptance/tolerance and paranoid ideation. In study 1, we examined the hypotheses crosssectionally based on questionnaire data from a combined sample $(N=125)$ consisting of people with a psychotic disorder, people at risk of developing psychosis, and healthy controls. In study 2, we examined the same hypotheses longitudinally by employing the experience sampling method in people with clinically relevant psychopathology below diagnostic threshold $(N=138)$.

Results In study 1, the association between modification and paranoid ideation was not moderated by comprehension or self-support. However, comprehension and self-support moderated the association between acceptance/tolerance and paranoid ideation. In study 2, the interaction effect between comprehension and acceptance/tolerance on paranoid ideation was confirmed.

Conclusion The results indicate that comprehending and accepting/tolerating emotions could be protective against paranoid ideation.
\end{abstract}

Keywords Delusions $\cdot$ Schizophrenia $\cdot$ Coping $\cdot$ Interactions of emotion regulation strategies $\cdot$ ESM

In an influential model of psychosis, Garety et al. (2001) postulated that negative affect has a crucial influence on the interpretation of internal and external experiences and thereby significantly contributes to paranoid ideation. In support of this model, elevated levels of negative affect have been found to predict paranoid ideation in longitudinal studies (Fowler et al. 2012; Freeman et al. 2012; Lincoln et al. 2017a) and in studies based on the experience sampling

Electronic supplementary material The online version of this article (https://doi.org/10.1007/s10608-020-10173-6) contains supplementary material, which is available to authorized users.

Martin F. Wittkamp

martin.wittkamp@uni-hamburg.de

1 Clinical Psychology and Psychotherapy, Institute of Psychology, Faculty of Psychology and Movement Sciences, Universität Hamburg, Von-Melle-Park 5, 20146 Hamburg, Germany method (ESM) in samples with different levels of symptom severity (Ben-Zeev et al. 2011; Krkovic et al. 2018, 2020; Thewissen et al. 2011; Thiery et al. 2014). Moreover, an experimental manipulation of negative affect was shown to be associated with increases in paranoid ideation in the general population (Lincoln et al. 2010a, b) and negative affect mediated the impact of a social stressor on paranoid ideation in patients with a psychotic disorder (Freeman et al. 2015). In the face of this evidence for negative affect as a predictor of paranoid ideation, it stands to reason that difficulties in the regulation of negative affect play an important role in the formation of paranoid ideation.

Emotion regulation has been defined as a process comprising the monitoring, appraisal and modification of the quality, intensity, and duration of affective states (Thompson 1994). It has further been conceptualised as a set of regulation strategies. These strategies are often subdivided into those considered maladaptive, such as suppression 
(Campbell-Sills et al. 2006) and rumination (Nolen-Hoeksema et al. 2008), and those considered adaptive, such as cognitive reappraisal (John and Gross 2004) and acceptance (Flaxman et al. 2010). The assumption behind this subdivision is that maladaptive strategies will be positively, and adaptive strategies will be negatively associated with psychopathology (Aldao 2012). Associations with psychopathology, however, have only been robustly shown for the putatively maladaptive strategies, whereas findings on the association between adaptive strategies and overall psychopathology are heterogeneous (Aldao et al. 2010). A similar pattern of findings is evident in studies investigating emotion regulation strategies in psychosis (for a meta-analytic review see Ludwig et al. 2019). Although studies report a positive association between maladaptive strategies and positive psychotic symptoms overall, there is rather high heterogeneity in findings for adaptive strategies with only some studies reporting a significant negative association between cognitive reappraisal and positive psychotic symptoms and most studies reporting no significant association (Ludwig et al. 2019). This pattern of findings does not accord with the effective reduction of psychotic symptoms by means of clinical interventions that focus on conveying adaptive strategies, such as cognitive reappraisal and acceptance/tolerance (Cramer et al. 2016; Eichner and Berna 2016; Louise et al. 2018; Opoka et al. 2018). To resolve this paradox, it is necessary to improve our understanding of the conditions under which the use of putatively adaptive strategies can unfold their positive effect on paranoid ideation.

One way to do this is by taking a wider perspective on the process of emotion regulation. In most of the existing questionnaire studies, researchers have focused on isolated, putatively adaptive emotion regulation strategies and especially on cognitive reappraisal (Ludwig et al. 2019) without taking into account possible interactions with other strategies. This approach may be too narrow, because evidence from experimental and ESM studies suggests that individuals use various emotion regulation strategies simultaneously to deal with emotions (for a review, see Ford et al. 2019). Beyond that, adaptive combinations of regulatory strategies have been found to outperform both single strategy use and sequences of ineffective multiple strategies (Aldao et al. 2015; Bonanno and Burton 2013; Heiy and Cheavens 2014). Thus, taking combinations of strategies into account rather than focusing on isolated strategies is more likely to help us identify the nature of emotion regulation relevant to mental health and well-being.

A model of emotion regulation that takes this type of approach by focussing on the effective interplay of strategies is the adaptive coping with emotions model (ACE) put forward by Berking and Whitley (2014). This model includes (1) awareness, (2) clarity, (3) understanding the cause of an emotion, and (4) providing compassionate self-support in handling an emotion. The model further discriminates between (5) a modificatory pathway, which includes modification strategies, such as cognitive reappraisal, positive refocusing and social sharing and (6) a non-modificatory pathway, which includes non-modificatory strategies, such as acceptance and tolerance. Awareness, clarity, and understanding of an emotion (1-3, which we will subsume as comprehension) and compassionate self-support (4, which we will shorten to self-support in the following) are stipulated to impact on the emotion regulation process indirectly by facilitating modification (5) and acceptance/tolerance (6; Berking and Whitley 2014). In support of this assumption, a cross-sectional study demonstrated the interaction of clarity and cognitive reappraisal to be negatively associated with PTSD symptoms (Boden et al. 2012) and an experimental study showed a moderating effect of self-support on the effect of cognitive reappraisal on depressive symptoms (Diedrich et al. 2016). Thus, taking these interactions into account might also inform our understanding of the nature of emotion regulation difficulties that drive psychotic symptoms, such as paranoid ideation.

This study aims to identify adaptive emotion regulation patterns that are negatively associated with and may be protective against paranoid ideation. We expect to find that comprehension and self-support will strengthen the negative association between paranoid ideation and modification (H1) and between paranoid ideation and acceptance/tolerance (H2). To ascertain the robustness of findings, the hypotheses were tested using data from two studies that focused on different levels of symptom severity and used different designs. Study 1 used a sample of people with psychotic disorders, a sample of participants with high risk to develop a psychotic disorder and healthy controls, while study 2 used a sample of people with clinically relevant psychopathology below diagnostic threshold. Study 1 used a cross-sectional design, while study 2 used a longitudinal design with ESM. Data from study 1 stems from of a larger project on emotion regulation in psychotic disorders (LI 1298/7-1). Study 2 uses baseline data from a project testing the effect of an emotion regulation training (Wittkamp and Lincoln 2020).

\section{Study 1}

\section{Recruitment, Procedure, and Design of the Original Project}

The original study included participants with psychotic disorders, anxiety disorders, those at risk of psychosis and healthy controls who were recruited via in- and outpatient clinics in Hamburg, advertisements in local newspapers, blackboards, and online platforms. Inclusion criteria were sufficient German language skills, an age between 18 and 
65 years, IQ $>85$, no current suicidality, no neurological disorders or dementia, no diagnosis of a bipolar disorder or substance dependence and the ability to provide informed consent. Participants completed questionnaires and neuropsychological tests and partook in a social exclusion paradigm and an emotion regulation paradigm on 2 separate days. The details of recruitment, selection criteria and study procedure have been published elsewhere (Lincoln et al. 2017b) along with the data related to the social exclusion paradigm. The project was approved by the Ethics Committee of the German Psychological Society.

\section{Method}

\section{Participants}

In this study, we utilized data from the participants with psychotic disorders (PD, $n=60)$, those at risk of psychosis (AR, $n=25$ ) and the healthy controls (HC, $n=40$ ) who had provided baseline questionnaire data on emotion regulation and paranoid ideation. PD fulfilled criteria for a psychotic disorder according to the Mini International Neuropsychiatric Interview (M.I.N.I.; Sheehan et al. 1998) at the time of assessment or during the last 2 years. Alternatively, if the last psychotic episode was longer than 2 years ago, PD needed to have experienced at least two psychotic episodes in total and presently report either at least two symptoms with mild severity (cut-off $\geq 3$ ) or at least one symptom with moderate severity (cut-off $\geq 4$ ) on the positive or negative symptom subscale of the Positive and Negative Syndrome Scale (PANSS; Kay et al. 1987). AR fulfilled at least one of three criteria for prodromal syndromes as defined in the Structured Interview for Prodromal Syndromes (SIPS; Miller et al. 2003) and did not fulfil criteria for PD. HC did not fulfil criteria for any present or past Axis I disorder according to M.I.N.I., had no first-degree relative with a psychotic disorder, were not taking any psychopharmacological medication, and did not fulfil AR criteria. Sample characteristics for the combined sample and subgroups are presented in Table 1.

\section{Measures Used for this Study}

Adaptive emotion regulation strategies were assessed with the German version of the Emotion Regulation Skills Questionnaire - Emotion Specific (ERSQ-ES; Ebert et al. 2013). The scale contains 12 items rated on a five-point Likert scale ( $0=$ "not at all" to $4=$ "always"), where the use of emotion regulation strategies is rated specific to emotions. To prevent potentially biased self-reports due to memory deficits that are typically found in the PD and AR subgroups (Brewer et al. 2005; Leavitt and Goldberg 2009), we used an interview format. Participants were asked to recall a situation in which they experienced a particular negative emotion (anxiety, anger, sadness, and shame) and were then instructed to rate to which extent they applied each of the regulatory strategies. The ERSQ-ES in its original structure with nine emotion-specific subscales was reported to have good validity and reliability (Ebert et al. 2013). In our analyses we

Table 1 Sample characteristics, means and standard deviations of emotion regulation strategies and paranoid ideation and comparisons between subgroups in study 1

\begin{tabular}{|c|c|c|c|c|c|c|c|}
\hline \multirow[t]{2}{*}{ Variable } & \multicolumn{6}{|l|}{ Study 1} & \multirow{2}{*}{$\begin{array}{l}\text { Study } 2 \\
\text { Subthreshold } \\
\text { sample }(n=138)\end{array}$} \\
\hline & $\begin{array}{l}\text { Psychosis group } \\
(n=60)\end{array}$ & $\begin{array}{l}\text { At risk group } \\
(n=25)\end{array}$ & $\begin{array}{l}\text { Healthy con- } \\
\text { trols }(n=40)\end{array}$ & $\begin{array}{l}\text { Combined sam- } \\
\text { ple }(n=125)\end{array}$ & $\begin{array}{l}\text { Statistics for univariate Com- } \\
\text { parisons }\left(F, \chi^{2}\right)\end{array}$ & $\begin{array}{l}\text { Games Howell } \\
\text { Post Hoc Tests }\end{array}$ & \\
\hline Age & 40.15 (11.66) & $34.72(14.05)$ & $40.03(10.78)$ & $39.02(12.00)$ & $F(2,122)=2.04 ; \mathrm{n} . \mathrm{s}$ & n.s & $36.33(12.12)^{\mathrm{e}}$ \\
\hline Gender (\%female) & $63 \%$ & $72 \%$ & $68 \%$ & $66 \%$ & $\chi^{2}=0.63, d f=2 ; \mathrm{n} . \mathrm{s}$ & n.s & $75 \%{ }^{\mathrm{e}}$ \\
\hline Education in years & $16.26(4.09)^{\mathrm{a}}$ & $16.48(7.68)^{\mathrm{b}}$ & $18.32(4.08)^{\mathrm{c}}$ & $16.98(5.00)^{\mathrm{d}}$ & $F(2,112)=2.06 ; \mathrm{n} . \mathrm{s}$ & n.s & $17.07(4.80)^{\mathrm{e}}$ \\
\hline Comprehension & $3.18(0.63)$ & $2.97(0.65)$ & $3.43(0.44)$ & $3.22(0.60)$ & $F(2,122)=5.1 ; p<0.01$ & $\mathrm{HC}>\mathrm{AR}(p<0.01)$ & $2.26(0.77)$ \\
\hline Self-Support & $2.58(0.84)$ & $2.11(0.85)$ & $2.97(0.84)$ & $2.62(0.89)$ & $F(2,122)=7.8 ; p<0.01$ & $\mathrm{HC}>\mathrm{PD}, \mathrm{AR}(p<0.001)$ & $2.17(0.95)$ \\
\hline Modification & $1.82(0.73)$ & $1.48(0.74)$ & $2.45(0.62)$ & $1.95(0.78)$ & $F(2,122)=16.96 ; p<0.001$ & $\mathrm{HC}>\operatorname{AR}(p<0.001)$ & $1.77(0.93)$ \\
\hline Acc/Tol & $2.36(0.81)$ & $2.12(0.70)$ & $2.96(0.56)$ & $2.51(0.79)$ & $F(2,122)=12.77 ; p<0.001$ & $\mathrm{HC}>\mathrm{PD}, \mathrm{AR}(p<0.001)$ & $2.34(0.81)$ \\
\hline Paranoid Ideation & $2.23(1.04)$ & $2.19(0.76)$ & $1.08(0.12)$ & $1.853(0.96)$ & $F(2,122)=27.55 ; p<0.001$ & $\mathrm{HC}<\mathrm{PD}, \mathrm{AR}(p<0.001)$ & $2.00(1.59)$ \\
\hline
\end{tabular}

Comprehension, Self-Support, Modification, Acceptance/Tolerance were measured with the Emotion Regulation Skills Questionnaire - Emotion Specific. Paranoid ideation was measured with the Paranoia Checklist 18 item version (study 1) and 3 item version (study 2)

Acc/Tol acceptance/tolerance, $\mathrm{HC}$ healthy controls, $\mathrm{PD}$ psychosis group, AR at risk group

${ }^{\mathrm{a}} \mathrm{n}=5$ missing cases

${ }^{\mathrm{b}} \mathrm{n}=3$ missing cases

${ }^{c} \mathrm{n}=2$ missing cases

${ }^{\mathrm{d}} \mathrm{n}=10$ missing cases

${ }^{e} \mathrm{n}=21$ missing cases 
focused on the following subscales: comprehension, selfsupport, modification, and acceptance/tolerance. Comprehension was calculated as the mean score of awareness, clarity and understanding and showed good internal consistency (Cronbach's alpha $=0.86$ ). Self-support, modification, and acceptance/tolerance also had acceptable internal consistencies across emotions (all Cronbach's alphas $>0.70$ ). Thus, we used the average score across emotions for each subscale.

Paranoid ideation was assessed with a German version of the frequency scale of the Paranoia Checklist (PCL-18; Freeman et al. 2005). The PCL-18 consists of 18 items that are self-rated on a five-point Likert scale ( 1 = "rarely", 2 = "once a month", 3 = "once a week", $4=$ "several times a week", $5=$ "at least once a day"). In this study we used the mean scores (range 1-5). The German version of the PCL-18 has shown excellent internal consistency and good convergent validity in clinical and non-clinical samples (Lincoln et al. 2010a, b). In this study, Cronbach's alpha of the total scale was 0.96 .

\section{Statistical Analyses}

Statistical analyses were conducted in SPSS (version 25) and were performed on the combined sample to represent different levels of paranoid ideation severity along the continuum of psychosis (see Table 1) and thereby to increase variance in paranoid ideation and test-power. As paranoid ideation was not normally distributed (skewness $=2.08, \mathrm{SE}=0.44$, and kurtosis $=4.56, \mathrm{SE}=0.088$ ), we applied bootstrapping based on 1000 samples. A multiple regression analysis with forced entry was conducted with paranoid ideation as the dependent variable and four strategies of emotion regulation (comprehension, self-support, modification, and acceptance/tolerance) along with their interactions as independent variables. Independent variables were centred around their grand mean. The interaction graphs were created based on predicted mean values calculated with the PROCESS macro (Hayes 2017). We found the missing data in the emotion regulation assessments $(0.6 \%)$ to be missing completely at random according to Little's MCAR test, $\chi^{2}(409)=454.07$, $p=0.061$. We therefore used the expectation maximization algorithm (Dempster et al. 1977) for imputation.

\section{Results}

\section{Descriptive Statistics}

Table 1 provides an overview of descriptive statistics of emotion regulation and paranoid ideation in each subgroup and the combined sample and statistical comparisons between subgroups. HC had significantly lower levels of paranoid ideation than AR and PD. AR and PD did not differ in this regard.

\section{Regression Analysis for the Association of Emotion Regulation Strategies and Paranoid Ideation}

Hypothesis 1: As shown in Table 2, modification was negatively associated with paranoid ideation, but this association was not moderated by comprehension or self-support.

Hypothesis 2: There were significant interaction effects of acceptance/tolerance and comprehension as well as acceptance/tolerance and self-support on paranoid ideation (see Table 2). As can be seen in Fig. 1a, paranoid ideation was lower for individuals who were high in acceptance/tolerance who also had high comprehension of their emotions as well, in comparison to those who were high in acceptance/ tolerance but low in comprehension. Statistically, this was significant at $t(124)=3.64, p<0.001, d=0.33$. More precisely, the interaction accounted for an approximate 1-point difference in the mean rating of the frequency of paranoid ideation that varied from 1 (rarely) to 5 (at least once a day). Paranoid ideation was comparably low for individuals who were low in both acceptance/tolerance and comprehension, compared to individuals who scored high on both strategies. Figure $1 \mathrm{~b}$ shows that the paranoid ideation score was lower for individuals who had low acceptance/tolerance but scored high on self-support than for those who were high in both acceptance/tolerance and self-support; $t(124)=3.49$, $p<0.001, d=0.31$. Finally, participants who had low acceptance/tolerance but high self-support had lower paranoid ideation than individuals who scored low on both strategies; $t(124)=3.86, p<0.001, d=0.35$. This difference was illustrated by a 1-point mean difference in the mean rating of the frequency of paranoid ideation that varied from 1 (rarely) to 5 (at least once a day).

\section{Additional Analyses}

Due to a relatively high proportion of female participants in our sample, we conducted a reanalysis controlling for gender (see Table A1 in the Online Appendix). In this analysis, modification was no longer directly associated with paranoid ideation.

To assure the robustness of our findings using the imputed data, we further repeated the calculations constraining the analysis to complete cases. There were no differences between imputed and complete case results (see Table A2 in the Online Appendix). 
Table 2 Study 1: multiple regression analysis for emotion regulation strategies as independent variables and paranoid ideation as dependent variable

\begin{tabular}{lrccc}
\hline Variable & \multicolumn{1}{l}{ B } & SE & $95 \% \mathrm{CI}^{\mathrm{a}}$ & $p$ \\
\hline Modification & -0.35 & 0.15 & {$[-0.67,-0.05]$} & 0.028 \\
Acceptance/tolerance & 0.06 & 0.16 & {$[-0.22,0.38]$} & 0.677 \\
Self-support & -0.11 & 0.14 & {$[-0.44,0.13]$} & 0.407 \\
Comprehension & -0.08 & 0.21 & {$[-0.45,0.36]$} & 0.724 \\
Modification $\times$ self-support & -0.07 & 0.22 & {$[-0.47,0.46]$} & 0.746 \\
Modification $\times$ comprehension & 0.21 & 0.39 & {$[-0.75,0.87]$} & 0.623 \\
Acceptance/tolerance $\times$ self-support & 0.48 & 0.20 & {$[0.01,0.82]$} & 0.019 \\
Acceptance/tolerance $\times$ comprehension & -0.76 & 0.32 & {$[-1.30,-0.04]$} & 0.019 \\
\hline
\end{tabular}

CI confidence interval

$\mathrm{R}^{2}=0.20$

${ }^{\mathrm{a} C}$ Confidence interval per bootstrap with 1.000 samples

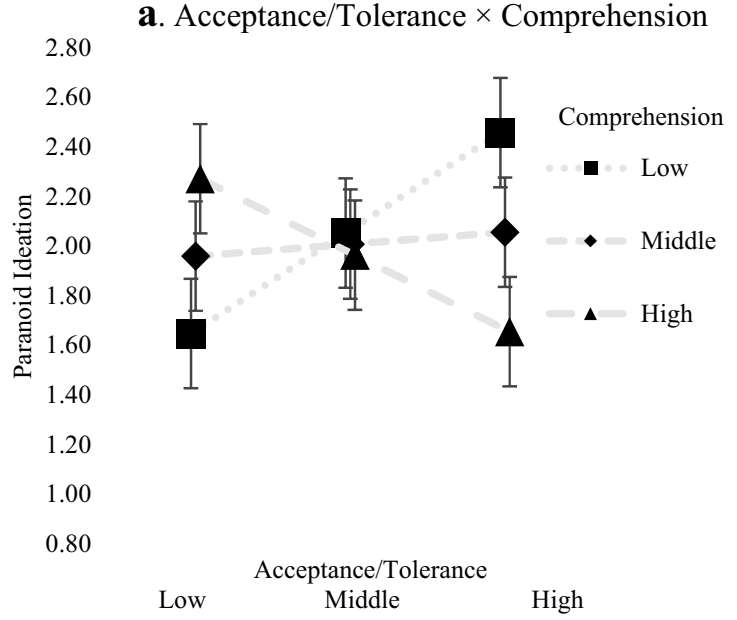

Fig. 1 Interaction effects between acceptance/tolerance and comprehension (a) and acceptance/tolerance and self-support (b). Predicted level of paranoid ideation (PCL-18) for combinations of

\section{Study 2}

\section{Recruitment, Procedure, and Design of the Original Project}

Participants were recruited via online platforms, newsletters, leaflets, and posters in public spaces in Hamburg, Germany. Inclusion criteria were sufficient German language skills, an age between 18 and 65 years and the presence of clinically relevant, subthreshold psychopathology in at least one symptom domain (positive psychotic, negative psychotic symptoms, depressive, anxiety or phobic symptoms) indicative of an increased risk to develop a psychotic disorder (for theoretical reviews on the transdiagnostic expression of risk to develop psychotic disorders, see Fusar-Poli et al. 2014; Van Os and Reininghaus 2016). b. Acceptance/Tolerance $\times$ Self-Support
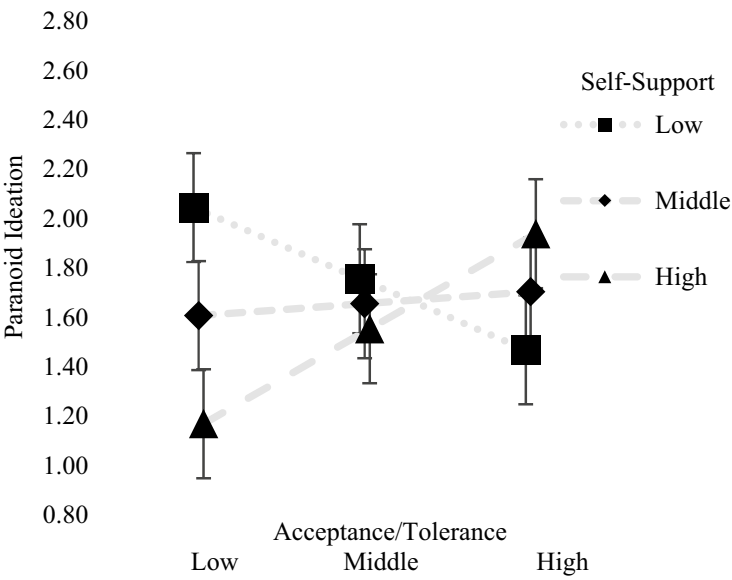

acceptance/tolerance and moderators (ERSQ-ES) at one $S D$ below, above and at the mean. Error bars indicate the standard error of the predicted mean. Lines only serve for better readability

Specific inclusion criteria were symptom frequency and distress deviating from the norm in any of the three subdomains of the Community Assessment of Psychic Experiences (CAPE; Stefanis et al. 2002: frequency $\geq 31$ and distress $\geq 24$ for positive psychotic symptoms, frequency $\geq 31$ and distress $\geq 26$ for negative psychotic symptoms, or frequency $\geq 17$ and distress $\geq 18$ for depressive symptoms) or in the anxiety or phobic subdomain of the Brief Symptom Inventory (BSI; Franke and Derogatis 2000: anxiety symptoms $\geq 0.68$ or phobic symptoms $\geq 0.39$ ). Exclusion criteria were current psychological treatment and the diagnosis of a current Axis I or Axis II disorder (specific phobia was not an exclusion criterion due to its high prevalence and relatively minor distress; Comer et al. 2011) as diagnosed with the Structured Clinical 
Interview for DSM-IV (SCID; German version: Wittchen et al. 1997).

The study was a two-armed, randomized-controlled trial comparing an 8-week emotion regulation group training to self-help bibliotherapy. Participants were pre-screened for eligibility via online-questionnaires and followed-up by a face-to-face assessment including diagnoses assessment. Before being allocated to the intervention or the control group participants underwent a baseline assessment that included a socio-demographic assessment, clinical questionnaires, and a 1-week ESM assessment of negative emotions, emotion regulation, and paranoid ideation. In this study, we used the ESM data on negative emotions, emotion regulation, and paranoid ideation from the combined intervention and control group. A detailed description of the procedure and data on the efficacy of the intervention will be published elsewhere (Wittkamp and Lincoln 2020). The study was approved by the local Ethics Committee of the University of Hamburg.

\section{Method}

\section{Participants}

The final sample consisted of $N=138$ participants. Sample characteristics are presented in Table 1. After the baseline ESM assessment but before baseline questionnaires, 21 participants dropped out from the study, resulting in missing data for age, gender, and years of education for these participants.

\section{ESM}

The ESM was administered via movisens XS Version 1.5.0 (movisens GmbH, Karlsruhe, Germany) on participants' private smartphones or on the study-smartphones. Participants were provided with a detailed explanation and examples of items along with an instruction sheet. Within the ESM assessment, over a period of six consecutive days, five times per day between 9 am and $10 \mathrm{pm}$, participants were notified with signal contingent, random time sampling (Wheeler and Reis 1991) with minimum time gaps of $1.5 \mathrm{~h}$ in between notifications. At each notification, participants were first asked to rate the intensity of each of a pre-defined list of negative emotions experienced just before the notification, then to select the negative emotion with the highest intensity just before the notification and to indicate the emotion regulation strategies they had used for that negative emotion. This was followed by a brief assessment of paranoid ideation experienced just before the notification.

\section{Measures Used in the ESM}

The negative emotions anxiety, sadness, anger, shame, insecurity, and loneliness were each rated in their intensity on an 11-point self-report Likert scale $(0=$ "not at all" to $10=$ "very strong"). The scale demonstrated good internal consistency between individuals (Cronbach's alpha $=0.84$ ) so we used the mean score of all negative emotions as a coefficient of negative emotions.

Adaptive emotion regulation strategies were assessed with the German version of the ERSQ-ES (Ebert et al. 2013) that we adapted for the ESM. At each notification, participants reported the use of regulatory strategies on a five-point Likert scale $(0=$ "does not apply at all" to $4=$ "fully applies") specific to the emotion that they had rated as most intensive (anxiety, sadness, anger, shame, insecurity, or loneliness). The 12 items used in the original form of the ERSQ-ES were slightly adapted to ensure that they referred to the emotion experienced and the regulation strategy applied before the notification (e.g., modification: In the situation just before the beep, I was able to influence my anxiety). Comprehension was calculated as the mean value of awareness, clarity, understanding and demonstrated excellent internal consistency between individuals (Cronbach's alpha $=0.90$ ). Selfsupport, modification, and acceptance/tolerance, in our sample, also showed good internal consistency between individuals and across emotions (all Cronbach's alphas $>0.88$ ). Thus, we used the individual subscales averaged across emotions.

Paranoid ideation was assessed with the German, threeitem version of the PCL (PCL-3; Schlier et al. 2016). The included items ("I need to be on my guard against others", "People try to upset me", and "Strangers and friends look at me critically") have been shown to be valid and change-sensitive (Schlier et al. 2016). Items were rated on an 11-point Likert scale $(0=$ "not at all", $10=$ "very much"). Due to nonnormality of the data, the scale was dichotomized to presence $(=1)$ or absence $(=0)$ of paranoid ideation.

\section{Statistical Analyses}

Statistical analyses were run with IBM SPSS Statistics (version 25). To account for the hierarchical data structure, we conducted time-lagged multilevel binomial probit regression analyses with random intercept and random slopes. Independent variables (comprehension, self-support, modification, and acceptance/tolerance) were centered around the person mean. To allow for chronological inferences regarding the association between emotion regulation and paranoid ideation, we used measurements of emotion regulation at a given time-point $(\mathrm{t})$ to predict paranoid ideation at the following measurement point $(t+1)$. To ensure that emotions that required regulation were 
present, solely measurements with negative emotions $>0$ at $t$ were included. Overnight lags were excluded. To test whether emotion regulation at $t$ had an impact on paranoid ideation at $t+1$ beyond the effect of preceding negative emotions and paranoid ideation, we controlled for negative emotions and paranoid ideation at t. For the interaction graph, we used mean values calculated based on predicted probabilities for paranoid ideation, which were defined by combinations of comprehension and acceptance/tolerance each at three different levels: $<1$ SD below (=low), $>1$ SD above (=high) and between $>1$ SD below and $>1$ SD above (= middle) its mean (see Fig. 2).

\section{Results}

\section{Descriptive Statistics}

Three participants were excluded from the analyses due to insufficient compliance with the ESM ( $>90 \%$ missing data). After exclusion of these cases, the average compliance rate was $78.47 \%$ (range: $10-100 \%$ ). This corresponds to compliance rates found in other ESM studies (Silvia et al. 2013). Paranoid ideation was present on $48 \%$ of measurement points and negative emotions were present (intensity $>0$ ) on $80 \%$ of the measurement points.

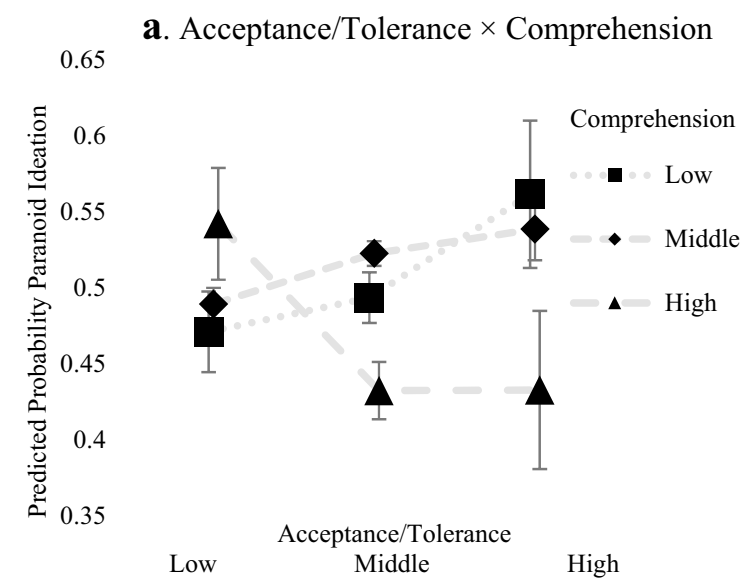

Fig. 2 Interaction effects between acceptance/tolerance and comprehension (a) that was significant, and acceptance/tolerance and self-support (b) that was not significant. Predicted probability of paranoid ideation (PCL-3) at $\mathrm{t}+1$ for measures with combinations of acceptance/tolerance and comprehension or self-support (ERSQ-
Emotion Regulation Strategies at $\mathbf{t}$ as Predictors of Paranoid Ideation at $t+1$ While Controlling for Paranoid Ideation and Negative Emotions at $t$

Results from the regression analysis can be seen in Table 3 . Modification at $t$ significantly predicted paranoid ideation at $t+1$. There was no significant relationship between acceptance/tolerance at $\mathrm{t}$ and paranoid ideation at $\mathrm{t}+1$.

Hypothesis 1: There were no significant interaction effects, neither between modification and comprehension at $\mathrm{t}$ nor between modification and self-support at $t$ on paranoid ideation at $\mathrm{t}+1$.

Hypothesis 2: The interaction of acceptance/tolerance and comprehension at $t$ predicted a decreased likelihood to experience paranoid ideation at $t+1$. As can be seen in Fig. 2a, the predicted probability for paranoid ideation at $t+1$ was lower when comprehension was moderate or high at $t$ and acceptance/tolerance was high at $t$ than it was when acceptance/tolerance was high and comprehension was low; $t$ $(85)=2.08, p<0.05$. $d=0.44$. Specifically, the chances of experiencing paranoid ideation at $\mathrm{t}+1$ were decreased by $13 \%$ when comprehension was moderate or high at t. There was no significant effect of interaction between acceptance/ tolerance at $t$ and self-support at $t$ on paranoid ideation at $\mathrm{t}+1$ (see Fig. 2b).

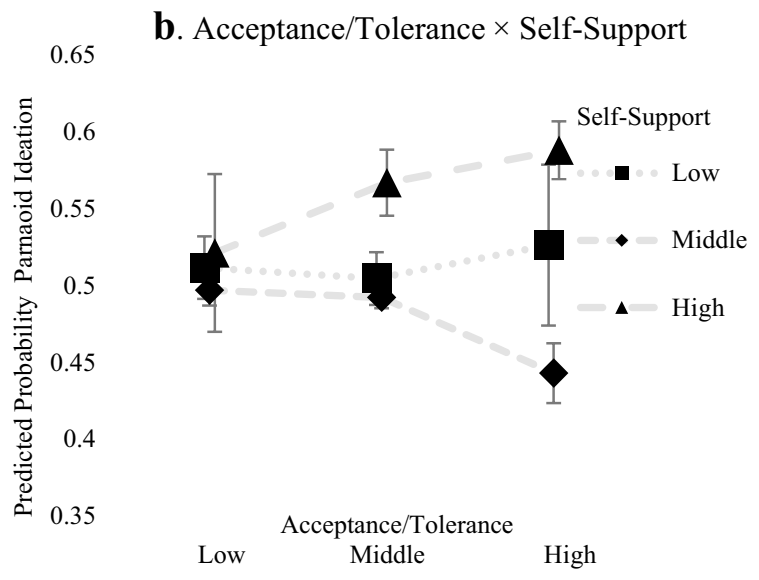

ES) $<1 S D$ below (=low), $>1$ SD above (=high) and between $>1$ $S D$ below and $>1 S D$ above (=middle) the mean value of comprehension, self-support, or acceptance/tolerance as measured at t. Error bars represent standard errors of the mean. Lines only serve for better readability 


\section{Additional Analyses}

To ascertain the robustness of our findings we did a reanalysis excluding four participants with less than $70 \%$ data available in the ESM. The main findings did not differ from those found in our original analysis in which we took we took the less conservative approach by excluding participants with less than $90 \%$ data available (see Table A3 in the Online Appendix).

\section{Discussion}

In the present study we investigated links between the interplay of adaptive emotion regulation strategies and paranoid ideation.

\section{Modification as a Predictor of Paranoid Ideation and Moderating Effects of Comprehension and Self-support}

We predicted that an adequate comprehension and selfsupport would strengthen the negative association between modification and paranoid ideation. However, in study 1 , there was a direct association between modification and paranoid ideation that was not moderated by comprehension or self-support. In study 2, modification predicted subsequent paranoid ideation and this effect was also not moderated by comprehension or self-support. Hence, although modification and paranoid ideation were negatively associated in both studies, this association was independent of other emotion regulation strategies. This is surprising, given the ACE model's assumption that comprehension of emotions and self-support lead to a more focused and targeted attempt to modify emotions (Berking and Whitley 2014) that has been partially confirmed in previous research (e.g. Boden et al. 2012; Diedrich et al. 2016; Kalokerinos et al. 2019). However, these previous studies have mostly tested moderating effects specifically for cognitive reappraisal rather than for modification in general, which might explain the diverging findings. Another explanation for not detecting moderating effects in both studies is that the nature of the association might be more complex than a mere moderation. Interestingly, Berking et al. (2012) found an indirect effect between comprehension, self-support, and psychopathology via modification. Thus, it is conceivable that comprehension and self-support constitute prerequisites for the application of modification. Taken together, cross-sectionally as well as longitudinally modification seems to be protective against paranoid ideation. However, the underlying mechanisms remain to be disentangled in future research.

\section{Acceptance/Tolerance as a Predictor of Paranoid Ideation and the Moderating Effect of Comprehension}

Our expectation that the association between acceptance/tolerance and paranoid ideation would be moderated by comprehension was confirmed. In study 1 , we found that participants with a strong ability to accept and tolerate, along with a better understanding of their emotions, reported considerably less paranoid ideation than those who were high in acceptance and tolerance but reported low understanding of their emotions. The finding from study 2 confirmed this pattern by showing that when participants adequately accepted and understood their emotions, the likelihood of experiencing subsequent paranoid ideation was lower than when participants accepted their emotion without adequately understanding it. This is in line with what we had expected based on the ACE model. These observations further corroborate previous experimental research finding participants with lower baseline levels of comprehension, acceptance,
Table 3 Study 2: binary multilevel probit regression analysis with emotion regulation strategies at $t$ as independent variables and paranoid ideation at $\mathrm{t}+1$ as dependent variable

\begin{tabular}{lrlll}
\hline Variable & \multicolumn{1}{l}{$B$} & SE & $95 \%$ CI & $p$ \\
\hline Modification & 0.12 & 0.05 & {$[0.03,0.20]$} & 0.012 \\
Acceptance/tolerance & -0.03 & 0.05 & {$[-0.12,0.07]$} & 0.588 \\
Self-support & 0.03 & 0.04 & {$[-0.04,0.11]$} & 0.387 \\
Comprehension & -0.08 & 0.04 & {$[-0.16,-0.01]$} & 0.036 \\
Modification $\times$ self-support & -0.08 & 0.06 & {$[-0.18,0.04]$} & 0.187 \\
Modification $\times$ comprehension & -0.05 & 0.06 & {$[-0.17,0.07]$} & 0.433 \\
Acceptance/tolerance $\times$ self-support & 0.00 & 0.05 & {$[-0.09,0.10]$} & 0.946 \\
Acceptance/tolerance $\times$ comprehension & -0.12 & 0.06 & {$[-0.23,-0.01]$} & 0.037 \\
Negative emotions & 0.08 & 0.04 & {$[0.01,0.16]$} & 0.033 \\
Paranoid ideation & 0.16 & 0.09 & {$[-0.00,0.33]$} & 0.056 \\
\hline
\end{tabular}

Only cases were included with negative emotions $>0$ at $\mathrm{t}$. Negative emotions and paranoid ideation at $\mathrm{t}$ were included as control variables

$C I$ confidence interval 
and tolerance to be more likely to respond to a stressor with an increase of paranoid ideation (Lincoln et al. 2015). Extending this finding, both of our studies showed that high acceptance without an adequate understanding of emotions was associated with increased paranoid ideation. This suggests that acceptance is only adaptive when it is applied in combination with an adequate understanding of the respective emotion.

\section{Acceptance/Tolerance as a Predictor of Paranoid Ideation and the Moderating Effect of Self-support}

In study 1, we found self-support to moderate the association between acceptance/tolerance and paranoid ideation. Specifically, paranoid ideation was low when self-support was high and acceptance/tolerance was low. In study 2, self-support did not moderate the association between acceptance/tolerance and paranoid ideation. Thus, the present results do not corroborate the theoretical assumptions from the ACE model concerning self-support (Berking and Whitley 2014). However, findings from study 1 are in line with an experimental study by Diedrich et al. (2014) demonstrating that in direct comparison, acceptance and self-support were equally effective at down-regulating emotions. Our findings complement this study by indicating that self-support might be adaptive when applied as an alternative regulatory strategy by those individuals who do not accept their emotions. This inference from study 1 might further help to explain our unexpected finding that paranoid ideation was relatively low when acceptance/tolerance and comprehension were low. Specifically, we might speculate that self-support compensated for a lack of acceptance and thereby lowered levels of paranoid ideation. In sum, contrary to what we expected, rather than strengthening the negative association between acceptance and tolerance with paranoid ideation, self-support seemed to serve as an alternative strategy associated with decreased paranoid ideation for those participants who did not accept their emotions. However, this finding was not confirmed longitudinally and therefore needs to be reexamined in future research.

\section{General Discussion}

Interestingly, our findings were different for modification and acceptance. We found a direct negative effect of modification on paranoid ideation, irrespective of how well an emotion was understood. For acceptance we found that it only negatively influenced paranoid ideation provided that an emotion was well understood. A closer examination of the constructs of modification and acceptance could help to explain these diverging findings. Modification describes the ability to adaptively change emotions (Berking and Whitley 2014). In this broad definition, modification includes a range of strategies that address factors associated with an emotion, such as an underlying problem (cf. problem-solving). Detailed information about an emotion might not be necessary for some of these strategies (e.g. problem-solving) to work. In contrast, acceptance refers to a willingness to stay in contact with the emotion itself (Campbell-Sills et al. 2006). Thus, it seems plausible that acceptance might be crucially facilitated when an emotion is well-defined and understood in contrast to a situation, in which the emotional state is diffuse. Possibly, the attempt to accept a diffuse emotional state results in a form of passive resignation (cf. Garnefski and Kraaij 2006). This could also help to explain our finding that acceptance in combination with an insufficient understanding leads to high levels of paranoid ideation.

\section{Limitations}

The present findings should be interpreted in the light of some limitations. First, in both studies, self-support was measured with only one item. Nevertheless, a validation study showed that the applied self-support assessment with the ERSQ-ES had good psychometric properties (Ebert et al. 2013), which was also confirmed by acceptable to good internal consistencies found in study 1 and study 2 . Second, in study 2 , we had to dichotomize paranoid ideation due to non-normality of the distribution and thereby relevant information may have been lost (Schlier et al. 2016). Third, PD and AR in study 1 included high percentages of female participants as compared to most at risk and psychosis samples (for a review, see Ochoa et al. 2012). This might limit the generalizability of our findings because gender differences have been found for emotion regulation (Nolen-Hoeksema 2012), which might affect the associations between regulatory strategies and paranoid ideation we examined here. Indeed, a reanalysis controlling for gender indicated that the direct association between modification and paranoid ideation could be specific to females, whereas adaptive patterns of acceptance and self-support or comprehension seem to hold, irrespective of gender. Finally, severe difficulties in awareness and clarity, which have been found in samples of patients with psychosis and those at risk of developing psychosis (Kimhy et al. 2012; Van Rijn et al. 2011), might have biased self-reports of discrete negative emotions in study 2 . Future studies could account for this by using an alternative classification of emotion, such as valence and arousal (Wilhelm and Schoebi 2007).

\section{Implications for Future Research and Clinical Practice}

Despite these limitations our findings corroborate the theoretical assumption that it is important to consider the interplay of adaptive emotion regulation strategies in order to 
understand better what is driving psychopathology (Aldao and Nolen-Hoeksema 2013; Berking and Whitley 2014; Ford et al. 2019). Specifically, our results suggest that accepting emotions can prevent subsequent paranoid ideation but only if emotions have been well comprehended. Our findings also suggest that self-support could be protective against paranoid ideation for those individuals who do not accept their emotions. Future research needs to clarify whether these adaptive patterns of emotion regulation are transdiagnositic or specific to paranoid ideation. Furthermore, it needs to be examined whether other adaptive emotion regulation patterns that are related to other psychopathology, such as PTSD or depressive sypmtoms (Boden et al. 2012; Diedrich et al. 2016), are also associated with paranoid ideation. It must be noted that this study along with the previous research investigates only one-directionally how emotion regulation strategies predict paranoid ideation. It is plausible however, that strategies emerge as a reaction to a present symptom and the accompanying negative emotion in an effort to reduce it. This could be an interesting question for future studies. Comprehension as a moderator is of special importance, given previous findings that people with a psychotic disorder and those at risk have lower levels of comprehension than healthy controls (Kimhy et al. 2012, 2016; Van Rijn et al. 2011). Our findings also have to be interpreted in the light of the theoretical assumption that negative emotion merely represents one of many factors that are assumed to precede paranoid ideation in cognitive models (Garety et al. 2001). Hence, it is not surprising that the associations we found were rather small. However, applying adaptive regulation patterns nevertheless accounted for clinically meaningful change in frequency of paranoid ideation and can further be well addressed in clinical interventions. Specifically, it could be promising to convey skills to become aware of, label and understand emotions to enable an adaptive and focused acceptance and tolerance. Finally, our findings suggest that individuals experiencing paranoid ideation could benefit from interventions that focus on effective combinations of regulation strategies, such as the Affect Regulation Training (Berking and Whitley 2014).

Acknowledgements We thank Johanna Sundag and Leonie Ascone Michelis for organizing the collection of data used for study 1. Furthermore, we thank the students who conducted the data collection.

Author Contributions Tania Lincoln and Martin Wittkamp contributed to the study conception and design. Material preparation and preparation of data collection were performed by Tania Lincoln and Martin Wittkamp. Data analyses were performed by Katarina Krkovic and Martin Wittkamp. The first draft of the manuscript was written by Martin Wittkamp and all authors contributed to the manuscript. All authors read and approved the final manuscript.

Funding Open Access funding enabled and organized by Projekt DEAL. The research in study 1 was funded by the German Research
Foundation, grant number DFG Li 1298/7-1. Research in study 2 did not receive any specific grant from fundieng agiencies in the public, commercial, or not-for-profit-sectors.

Data Availability Datasets described in this paper are available from the corresponding author on request.

\section{Compliance with Ethical Standards}

Conflict of Interest Martin F. Wittkamp, Katarina Krkovic and Tania M. Lincoln declare that they have no conflict of interest.

Ethical Approval This research was performed in line with the principles of the Declaration of Helsinki. Approval for study 1 was granted by the Ethics Committee of the German Psychological Society and approval for study 2 was granted by the Ethics Committee of the University of Hamburg.

Informed Consent Informed consent was obtained from all individual participants included in both studies.

Animal Rights No animal studies were carried out by the authors for this article.

Open Access This article is licensed under a Creative Commons Attribution 4.0 International License, which permits use, sharing, adaptation, distribution and reproduction in any medium or format, as long as you give appropriate credit to the original author(s) and the source, provide a link to the Creative Commons licence, and indicate if changes were made. The images or other third party material in this article are included in the article's Creative Commons licence, unless indicated otherwise in a credit line to the material. If material is not included in the article's Creative Commons licence and your intended use is not permitted by statutory regulation or exceeds the permitted use, you will need to obtain permission directly from the copyright holder. To view a copy of this licence, visit http://creativecommons.org/licenses/by/4.0/.

\section{References}

Aldao, A. (2012). Emotion regulation strategies as transdiagnostic processes: A closer look at the invariance of their form and function. Revista de Psicopatologia y Psicologia Clinica, 17(3), 261-277. https://doi.org/10.5944/rppc.vol.17.num.3.2012.11843

Aldao, A., Nolen-Hoeksema, S., \& Schweizer, S. (2010). Emotionregulation strategies across psychopathology: A meta-analytic review. Clinical Psychology Review, 30(2), 217-237. https://doi. org/10.1016/j.cpr.2009.11.004

Aldao, A., \& Nolen-Hoeksema, S. (2013). One versus many: Capturing the use of multiple emotion regulation strategies in response to an emotion-eliciting stimulus. Cognition \& Emotion, 27(4), 753-760. https://doi.org/10.1080/02699931.2012.739998

Aldao, A., Sheppes, G., \& Gross, J. J. (2015). Emotion regulation flexibility. Cognitive Therapy and Research, 39(3), 263-278. https:// doi.org/10.1007/s10608-014-9662-4

Ben-Zeev, D., Ellington, K., Swendsen, J., \& Granholm, E. (2011). Examining a cognitive model of persecutory ideation in the daily life of people with schizophrenia : A computerized experience sampling study. Schizophrenia Bulletin, 37(6), 1248-1256. https ://doi.org/10.1093/schbul/sbq041

Berking, M., Poppe, C., Luhmann, M., Wupperman, P., Jaggi, V., \& Seifritz, E. (2012). Is the association between various 
emotion-regulation skills and mental health mediated by the ability to modify emotions? Results from two cross-sectional studies. Journal of Behavior Therapy and Experimental Psychiatry, 43(3), 931-937. https://doi.org/10.1016/j.jbtep.2011.09.009

Berking, M., \& Whitley, B. (2014). The adaptive coping with emotions model (ACE Model). Affect regulation training (pp. 19-29). New York: Springer. https://doi.org/10.1007/978-1-4939-1022-9_3.

Boden, M. T., Bonn-Miller, M. O., Kashdan, T. B., Alvarez, J., \& Gross, J. J. (2012). The interactive effects of emotional clarity and cognitive reappraisal in Posttraumatic Stress Disorder. Journal of Anxiety Disorders, 26(1), 233-238. https://doi.org/10.1016/j. janxdis.2011.11.007

Bonanno, G. A., \& Burton, C. L. (2013). Regulatory flexibility: An individual differences perspective on coping and emotion regulation. Perspectives on Psychological Science, 8(6), 591-612. https ://doi.org/10.1177/1745691613504116

Brewer, W. J., Francey, S. M., Wood, S. J., Jackson, H. J., Pantelis, C., Phillips, L. J., et al. (2005). Memory impairments identified in people at ultra-high risk for psychosis who later develop first-episode psychosis. American Journal of Psychiatry, 162(1), 71-78. https://doi.org/10.1176/appi.ajp.162.1.71

Campbell-Sills, L., Barlow, D. H., Brown, T. A., \& Hofmann, S. G. (2006). Effects of suppression and acceptance on emotional responses of individuals with anxiety and mood disorders. Behaviour Research and Therapy, 44(9), 1251-1263. https://doi. org/10.1016/j.brat.2005.10.001

Comer, J. S., Blanco, C., Hasin, D. S., Liu, S. M., Grant, B. F., Turner, J. B., \& Olfson, M. (2011). Health-related quality of life across the anxiety disorders. The Journal of Clinical Psychiatry, 72(1), 43. https://doi.org/10.4088/JCP.09m05094blu

Cramer, H., Lauche, R., Haller, H., Langhorst, J., \& Dobos, G. (2016). Mindfulness- and acceptance-based interventions for psychosis: A systematic review and meta-analysis. Global Advances in Health and Medicine, 5(1), 30-43. https://doi.org/10.7453/gahmj .2015 .083

Dempster, A. P., Laird, N. M., \& Rubin, D. B. (1977). Maximum likelihood from incomplete data via the EM algorithm. Journal of the Royal Statistical Society: Series B (Methodological), 39(1), 1-22. https://doi.org/10.1111/j.2517-6161.1977.tb01600.x

Diedrich, A., Grant, M., Hofmann, S. G., Hiller, W., \& Berking, M. (2014). Self-compassion as an emotion regulation strategy in major depressive disorder. Behaviour Research and Therapy, 58, 43-51.

Diedrich, A., Hofmann, S. G., Cuijpers, P., \& Berking, M. (2016). Self-compassion enhances the efficacy of explicit cognitive reappraisal as an emotion regulation strategy in individuals with major depressive disorder. Behaviour Research and Therapy, 82, 1-10. https://doi.org/10.1016/j.brat.2016.04.003

Ebert, D. D., Christ, O., \& Berking, M. (2013). Entwicklung und Validierung eines Fragebogens zur emotionsspezifischen Selbsteinschätzung emotionaler Kompetenzen (SEK-ES). Diagnostica, 59(1), 17-32. https://doi.org/10.1026/0012-1924/a000079

Eichner, C., \& Berna, F. (2016). Acceptance and efficacy of Metacognitive Training (MCT) on positive symptoms and delusions in patients with schizophrenia: A meta-analysis taking into account important moderators. Schizophrenia Bulletin, 42(4), 952-962. https://doi.org/10.1093/schbul/sbv225

Flaxman, P., Blackledge, J., \& Bond, F. (2010). Acceptance and commitment therapy: Distinctive features. Abingdon: Routledge.

Ford, B. Q., Gross, J. J., \& Gruber, J. (2019). Broadening our field of view: The role of emotion polyregulation. Emotion Review, 11(3), 197-208. https://doi.org/10.1177/1754073919850314

Fowler, D., Hodgekins, J., Garety, P., Freeman, D., Kuipers, E., Dunn, G., et al. (2012). Negative cognition, depressed mood, and paranoia: A longitudinal pathway analysis using structural equation modeling. Schizophrenia Bulletin, 38(5), 1063-1073. https://doi. org/10.1093/schbul/sbr019

Franke, G., \& Derogatis, L. (2000). BSI. brief sympton inventorydeutsche version. Göttingen: Beltz Test.

Freeman, D., Emsley, R., Dunn, G., Fowler, D., Bebbington, P., Kuipers, E., et al. (2015). The stress of the street for patients with persecutory delusions: A test of the symptomatic and psychological effects of going outside into a busy urban area. Schizophrenia Bulletin, 41(4), 971-979. https://doi.org/10.1093/schbul/sbu173

Freeman, D., Garety, P. A., Bebbington, P. E., Smith, B., Rollinson, R., Fowler, D., et al. (2005). Psychological investigation of the structure of paranoia in a non-clinical population. British Journal of Psychiatry, 186(5), 427-435. https://doi.org/10.1192/ bjp.186.5.427

Freeman, D., Stahl, D., Mcmanus, S., Meltzer, H., Brugha, T., Wiles, N., \& Bebbington, P. (2012). Insomnia, worry, anxiety and depression as predictors of the occurrence and persistence of paranoid thinking. Social Psychiatry and Psychiatric Epidemiology, 47(8), 1195-1203. https://doi.org/10.1007/s00127-011-0433-1

Fusar-Poli, P., Yung, A. R., McGorry, P., \& van Os, J. (2014). Lessons learned from the psychosis high-risk state: Towards a general staging model of prodromal intervention. Psychological Medicine, 44(1), 17-24. https://doi.org/10.1017/S0033291713000184

Garnefski, N., \& Kraaij, V. (2006). Relationships between cognitive emotion regulation strategies and depressive symptoms: A comparative study of five specific samples. Personality and Individual Differences, 40(8), 1659-1669. https://doi.org/10.1016/j. paid.2005.12.009

Garety, P. A., Kuipers, E., Fowler, D., Freeman, D., \& Bebbington, P. E. (2001). A cognitive model of the positive symptoms of psychosis. Psychological Medicine, 31(2), 189-195. https://doi. org/10.1017/S0033291701003312

Hayes, A. F. (2017). Introduction to mediation, moderation, and conditional process analysis: A regression-based approach. New York: Guilford Publications.

Heiy, J. E., \& Cheavens, J. S. (2014). Back to basics: A naturalistic assessment of the experience and regulation of emotion. Emotion, 14(5), 878. https://doi.org/10.1037/a0037231

John, O. P., \& Gross, J. J. (2004). Healthy and unhealthy emotion regulation: Personality processes, individual differences, and life span development. Journal of Personality, 72(6), 1301-1334. https:// doi.org/10.1111/j.1467-6494.2004.00298.x

Kalokerinos, E. K., Erbas, Y., Ceulemans, E., \& Kuppens, P. (2019). Differentiate to regulate: Low negative emotion differentiation is associated with ineffective use but not selection of emotionregulation strategies. Psychological Science, 30(6), 863-879. https ://doi.org/10.1177/0956797619838763

Kay, S. R., Fiszbein, A., \& Opler, L. A. (1987). The positive and negative syndrome scale (PANSS) for schizophrenia. Schizophrenia bulletin, 13(2), 261-276. https://doi.org/10.1093/schbul/13.2.261

Kimhy, D., Vakhrusheva, J., Jobson-Ahmed, L., Tarrier, N., Malaspina, D., \& Gross, J. J. (2012). Emotion awareness and regulation in individuals with schizophrenia: Implications for social functioning. Psychiatry Research, 200(2-3), 193-201. https://doi.org/10.1016/j.psychres.2012.05.029

Kimhy, D., Gill, K. E., Brucato, G., Vakhrusheva, J., Arndt, L., Gross, J. J., \& Girgis, R. R. (2016). The impact of emotion awareness and regulation on social functioning in individuals at clinical high risk for psychosis. Psychological Medicine, 46(14), 2907-2918. https://doi.org/10.1017/S0033291716000490

Krkovic, K., Clamor, A., Schlier, B., \& Lincoln, T. M. (2020). Emotions and persecutory ideation in daily life: On the trail of the "chicken and egg" problem. Journal of Abnormal Psychology, 129(2), 215-223. https://doi.org/10.1037/abn0000495

Krkovic, K., Schlier, B., \& Lincoln, T. (2018). An experience sampling study on the nature of the interaction between traumatic 
experiences, negative affect in everyday life, and threat beliefs. Schizophrenia Research, 201, 381-387. https://doi. org/10.1016/j.schres.2018.05.030

Leavitt, V. M., \& Goldberg, T. E. (2009). Episodic memory in schizophrenia. Neuropsychology Review, 19(3), 312-323. https://doi. org/10.1007/s11065-009-9107-0

Lincoln, T. M., Ziegler, M., Lüllmann, E., Müller, M. J., \& Rief, W. (2010a). Can delusions be self-assessed? Concordance between self- and observer-rated delusions in schizophrenia. Psychiatry Research, 178(2), 249-254. https://doi.org/10.1016/j.psych res.2009.04.019

Lincoln, T. M., Lange, J., Burau, J., Exner, C., \& Moritz, S. (2010b). The effect of state anxiety on paranoid ideation and jumping to conclusions. An experimental investigation. Schizophrenia Bulletin, 36(6), 1140-1148. https://doi.org/10.1093/schbul/sbp029

Lincoln, T. M., Hartmann, M., Köther, U., \& Moritz, S. (2015). Dealing with feeling: Specific emotion regulation skills predict responses to stress in psychosis. Psychiatry Research, 228(2), 216-222. https://doi.org/10.1016/j.psychres.2015.04.003

Lincoln, T. M., Marin, N., \& Jaya, E. S. (2017a). Childhood trauma and psychotic experiences in a general population sample: A prospective study on the mediating role of emotion regulation. European Psychiatry, 42, 111-119. https://doi.org/10.1016/j. eurpsy.2016.12.010

Lincoln, T. M., Sundag, J., Schlier, B., \& Karow, A. (2017b). The relevance of emotion regulation in explaining why social exclusion triggers paranoia in individuals at clinical high risk of psychosis. Schizophrenia Bulletin, 44(4), 757-767. https://doi. org/10.1093/schbul/sbx135

Louise, S., Fitzpatrick, M., Strauss, C., Rossell, S. L., \& Thomas, N. (2018). Mindfulness- and acceptance-based interventions for psychosis: Our current understanding and a meta-analysis. Schizophrenia Research, 192, 57-63. https://doi.org/10.1016/j. schres.2017.05.023

Ludwig, L., Werner, D., \& Lincoln, T. M. (2019). The relevance of cognitive emotion regulation to psychotic symptoms-a systematic review and meta-analysis. Clinical Psychology Review. https://doi.org/10.1016/j.cpr.2019.101746

Miller, T. J., McGlashan, T. H., Rosen, J. L., Cadenhead, K., Ventura, J., McFarlane, W., et al. (2003). Prodromal assessment with the structured interview for prodromal syndromes and the scale of prodromal symptoms: Predictive validity, interrater reliability, and training to reliability. Schizophrenia Bulletin, 29(4), 703715. https://doi.org/10.1093/oxfordjournals.schbul.a007040

Nolen-Hoeksema, S. (2012). Emotion regulation and psychopathology: The role of gender. Annual Review of Clinical Psychology, 8(1), 161-187. https://doi.org/10.1146/annurev-clinpsy-03251 $1-143109$

Nolen-Hoeksema, S., Wisco, B. E., \& Lyubomirsky, S. (2008). Rethinking rumination. Perspectives on Psychological Science, 3(5), 400-424. https://doi.org/10.1111/j.1745-6924.2008.00088 . $\mathrm{X}$

Ochoa, S., Usall, J., Cobo, J., Labad, X., \& Kulkarni, J. (2012). Gender differences in schizophrenia and first-episode psychosis: A comprehensive literature review. Schizophrenia Research and Treatment, 2012, 1-9. https://doi.org/10.1155/2012/916198

Opoka, S. M., Ludwig, L., \& Lincoln, T. M. (2018). A systematic review of trials targeting depression and anxiety in patients with delusions. Zeitschrift für Psychologie, 226, 142-151. https://doi. org/10.1027/2151-2604/A000331

Schlier, B., Moritz, S., \& Lincoln, T. M. (2016). Measuring fluctuations in paranoia: Validity and psychometric properties of brief state versions of the Paranoia Checklist. Psychiatry Research, 241, 323-332. https://doi.org/10.1016/j.psychres.2016.05.002

Sheehan, D. V., Lecrubier, Y., Sheehan, K. H., Amorim, P., Janavs, J., Weiller, E., et al. (1998). The mini-international neuropsychiatric interview (M.I.N.I.): The development and validation of a structured diagnostic psychiatric interview for DSM-IV and ICD-10. The Journal of clinical psychiatry, 59(Suppl 20), 22-57.

Silvia, P. J., Kwapil, T. R., Eddington, K. M., \& Brown, L. H. (2013). Missed beeps and missing data. Social Science Computer Review, 31(4), 471-481. https://doi.org/10.1177/0894439313 479902

Stefanis, N. C., Hanssen, M., Smirnis, N. K., Avramopoulos, D. A., Evdokimidis, I. K., Stefanis, C. N., et al. (2002). Evidence that three dimensions of psychosis have a distribution in the general population. Psychological Medicine, 32(2), 347-358. https:// doi.org/10.1017/S0033291701005141

Thewissen, V., Bentall, R. P., Oorschot, M., \& Myin-germeys, I. (2011). Emotions, self-esteem, and paranoid episodes: An experience sampling study. British Journal of Clinical Psychology, 50(2), 178-195. https://doi.org/10.1348/014466510X508677

Thiery, E., Os, J. V., Myin-Germeys, I., Wichers, M., Kramer, I., Simons, C. J. P., et al. (2014). Time-lagged moment-to-moment interplay between negative affect and paranoia: New insights in the affective pathway to psychosis. Schizophrenia Bulletin, 40(2), 278-286. https://doi.org/10.1093/schbul/sbs 194

Thompson, R. A. (1994). Emotion Regulation: A theme in search of definition. Monographs of the Society for Research in Child Development, 593(2), 25-52.

Van Os, J., \& Reininghaus, U. (2016). Psychosis as a transdiagnostic and extended phenotype in the general population. World Psychiatry, 15(2), 118-124. https://doi.org/10.1002/wps.20310

Van Rijn, S., Schothorst, P., Wout, M. V., Sprong, M., Ziermans, T., van Engeland, H., et al. (2011). Affective dysfunctions in adolescents at risk for psychosis: Emotion awareness and social functioning. Psychiatry Research, 187(1-2), 100-105. https:// doi.org/10.1016/j.psychres.2010.10.007

Wheeler, L., \& Reis, H. T. (1991). Self-recording of everyday life events: Origins, types, and uses. Journal of Personality, 59(3), 339-354. https://doi.org/10.1111/j.1467-6494.1991.tb00252.x

Wilhelm, P., \& Schoebi, D. (2007). Assessing mood in daily life: Structural validity, sensitivity to change, and reliability of a short-scale to measure three basic dimensions of mood. European Journal of Psychological Assessment, 23(4), 258-267. https://doi.org/10.1027/1015-5759.23.4.258

Wittchen, H.-U., Zaudig, M., \& Fydrich, T. (1997). SKID. Strukturiertes Klinisches Interview für DSM-IV. Achse I und II. Handanweisung. Boston: Hogrefe Verlag für Psychologie.

Wittkamp, M. F., \& Lincoln, T. M. (2020). Efficacy of the affect regulation training in a sample with clinically relevant psychopathology below diagnostic threshold: A randomized controlled trial [Unpublished manuscript]. Clinical Psychology and Psychotherapy, Institute of Psychology, Universität Hamburg.

Publisher's Note Springer Nature remains neutral with regard to jurisdictional claims in published maps and institutional affiliations. 